ON

\title{
SOME OF THE CYCLICAL CHANGES
}

\author{
IN THE \\ H U M A N S Y S T E M
}

CONNECTED WITH SEASON.

BY

EDWARD SMITH, M.D., LL.B. LoND., L.R.C.P., ASSISTANT-PHYSICIAN TO THE HOSPITAL FOR CONSUMPTION AND DISEASES OF THE CHEST, BROMPTON; ETC.

Received Jan. 11th.-Read Feb. 22d, 1859.

I HAVE occupied the greater part of the last four years in an endeavour to determine the cyclical changes in the chief function of the body, viz., the respiration, and have, to some extent, succeeded in my object. Two papers have been published in the 'Transactions of the Royal Medical and Chirurgical Society' for 1856, in which I endeavoured to show the cyclical changes of the twenty-four hours, hour by hour, so far as relates to the rate of pulsation and respiration in health and in phthisis ; and a paper showing the variations in certain phenomena during the period of a month in the summer season, was published in the 'British and Foreign Medico-Chirurgical Review' in April of the same year. Since their publication $I$ have ascertained the hourly changes in the quantity of air inspired and the 
carbonic acid expired, with other phenomena of respiration, and have shown that the changes in the two latter subjects of inquiry are very similar to those of the rate of these functions, and that the progression of the day is as follows. Commencing in the depth of the night, at 1 to 3 a.m., when the function is at the lowest point of the twenty-four hours, there is a gradual and slow increase up to the usual period of rising, when it is at the lowest point of the daylight day-a point representing 75 per cent. of the whole average action of the twenty-four hours. After each meal, during the day, there is a rapid and progressive increase during about two and a half hours, to a maximum of about 35 per cent. of the quantity before breakfast, and then a more rapid decline, until the minimum of the daylight day is again obtained shortly before each meal. In the evening, however, there is seldom any increase after the supper; but whatever may be the existing circumstances, the changes diminish progressively, and at length fall very rapidly, until the minimum of the twenty-fours (viz., half of the maximum) is again attained. Thus, there is an unintermitting cycle of changes through the day and night coexistent with various physical phenomena, but not altogether explicable by them, in which the excess of action of the day alternates with an equal defect through the night.

I have now extended the same inquiry to the changes of the system in health from day to day, and month to month, through the cycle of the seasons, and have found, that however great and important the changes of the day may be in reference to the maintenance of the health and the fulfilment of the duties of the body, those of season are even more so, since they enable us to obtain a glimpse at some of the fundamental laws of the system. In presenting to the Society a short account of the results obtained, I purpose to avoid, as far as possible, extended physiological detail, and to limit myself to a short statement of the results and the deductions which flow from them.

Historical sketch.-Although the inquiry in the serial 
and extended form now mentioned is new, we have obtained from observers in physiology and practical medicine a large amount of knowledge which bears upon this subject. Thus, all physiological observers have noticed that the respiratory changes were never the same on any succeeding days. Vierordt has proved that they vary inversely, as the temperature and height of the barometer. Lavoisier and Liebig have both asserted that this function is less active in summer than in winter, partly because with heat the volume of air is increased, and thereby less oxygen remains in a given volume (omitting, however, all reference to the whole amount of air which is inspired), partly because the necessity for it to maintain the animal heat is lessened, and partly because it had been observed that the quantity of food taken was diminished. In my former inquiries I showed that the rate of respiration was lessened, and that of pulsation increased, with increasing temperature. It is believed, that in hot climates the respiratory function is lessened, whilst the function of the liver is increased in activity, and the diseases of those climates refer to the latter rather than to the former organ. Languor and relaxation are familiarly believed to prevail more in hot seasons and climates than in cold; and change of the weather towards the opposite, and therefore in all directions during the cycle of the seasons, is matter of common congratulation. Diseases vary in intensity, type, and frequency with season and climate, and even the action of remedies is believed to vary under those circumstances. Moreover, certain diseases are almost limited to certain seasons, and the various seasons as they complete their cycle bring with them causes of special liability to the production of disease. Certain animals gain weight in summer and lose it in winter, and the same fact has very recently been proved to occur in the prisoners at the convict gaol at Wakefield, where, for a series of ten years (as has been shown by the surgeon, Mr. Milner), they gain weight from May to September inclusive, and lose weight during the other months of the year-the diet and labour being the same throughout the year, and 
the periods of change being abrupt in April and October. Hence, without entering at length into the literature of this subject, it may be affirmed that much knowledge has been gained, and that what is lacking is chiefly in extent and also in demonstration on subjects on which we have as yet only opinions and inferences, and the determination of the laws and conditions upon which these cyclical changes depend.

Description of the method and apparatus.-The inquiry which I shall now proceed to describe has been made upon myself, æt. 39, and a gentleman in perfect health, æt. 48the one of us bearing great heat well, and the other suffering much from it : it was commenced on March 31st, 1858, and has been continued on about one hundred and fifty occasions to the present time. Each experiment has been made under precisely similar and normal circumstances, viz., between 7 and 8 a.m., before breakfast, in the sitting posture, and in the absence of all bodily exertion and mental excitement. The experiments may, therefore, be compared with each other; and since the disturbing influence of disease, food, and exertion was avoided, the variations in the state of the system must be chiefly due to season.

The apparatus employed (See Diagram in 'Phil. Trans.,' 1859) was a spirometer, already described to the Society, to measure the inspired air, and an analytical apparatus, whereby all the vapour and the carbonic acid could be removed from the expired air during the act of expiration, and for any period, however long or short. The latter consisted of a gutta-percha box, divided into chambers, and each chamber into cells, in such a manner that each column of one cubic inch of air was made to traverse, in a given direction, a surface of seven hundred superficial inches. The floor of each chamber was covered with a solution of caustic potass of sp. gr. 1.27, in order to absorb the carbonic acid; and as the expired air was first dried before it entered this box, the vapour which it carried away at its exit was arrested 


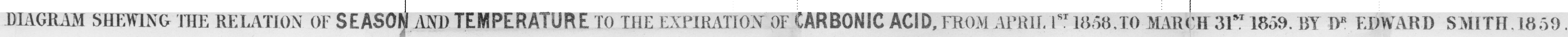

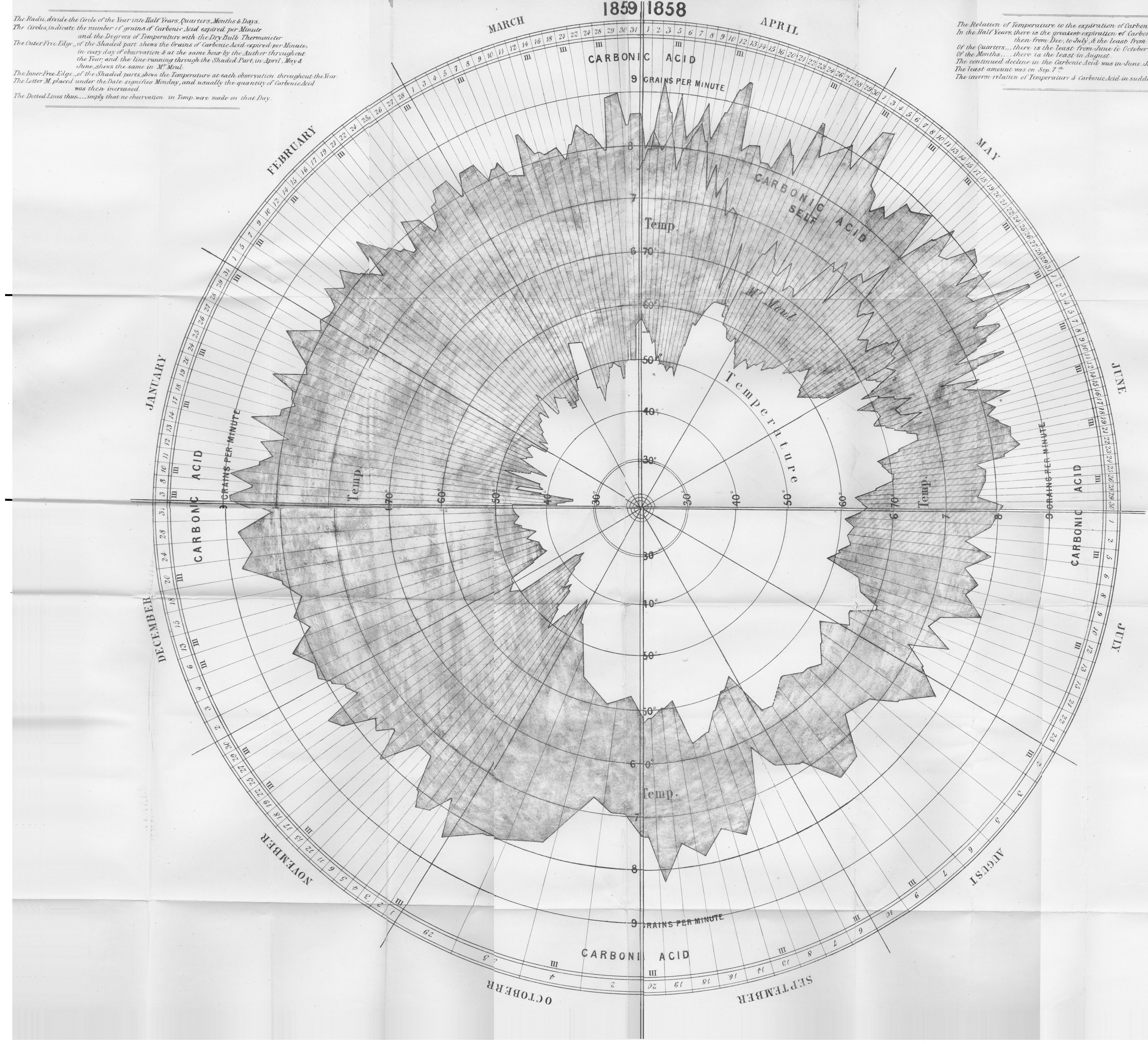


in a second drying apparatus. The addition to the weight of the box and the second drying apparatus gave the quantity of carbonic acid abstracted from the expired air. The balances employed were made by Oertling, and weigh to the $1^{\frac{1}{0} \sigma_{\text {th }}}$ of a grain, with 7 lbs. in each pan. There was no impediment to free expiration.

Results of the inquiry-The results which have been obtained are very decided, and the progression in the changes of the system are so uniform, and pursue a course so consonant with common and professional observation, that I think no valid objection can be taken to them. It is true that they have been derived from two persons onlybut these two belong to the two classes into which mankind may be divided in reference to the degree of tolerance of heat; and, although variations in period and degree may be found in others, it is very probable that the general progression which our returns show would still be applicable to others. It is this general progression in the cycle of changes, rather than the amount of change, which I think it important to demonstrate; and after having made some thousands of experiments more than any other observer has recorded, I have arrived at the opinion expressed by that laborious inquirer, Vierordt, that we can only obtain a large number of trustworthy observations from experiments long continued upon a few persons.

The general expression of the results obtained (see Diagram) is that, as the season advanced from spring to summer and autumn, all the respiratory phenomena were lessened, viz., the volume and much more the weight of air inspired, the amount of vapour and carbonic acid exhaled, the rate of respiration, the cooling of the body, and the muscular and vesicular actions of respiration. The highest state of the functions was in spring; the period of decline was the beginning of summer; the lowest state was towards the end of summer and in the beginning of autumn; and the period of increase was the beginning of winter. The highest months were April and May; the lowest were part 
of July, August, and part of September. The difference at these various periods was large and decided. Thus the extreme difference in myself between the highest and the lowest periods, when taken on the average of a month, was 30 per cent. of the larger quantity, in the quantity of air inspired, 32 per cent. in the rate of respiration, and 17 per cent. in the quantity of carbonic acid exhaled. In Mr. Moul, to whom I am indebted for having become the other subject for experiment, the diminution so early as the middle of June, when his experiments terminated, was 27 per cent. in both the carbonic acid expired and the air inspired, and 28 per cent. in the rate of respiration. He exhibited more quickly and in a greater degree than myself the effects of season at this comparatively early period, in accordance with an observation which $I$ published in connexion with former inquiries, viz., that those who suffer much from heat have an excess of all the seasonal changes. Hence season acts with varying power and rapidity upon different persons.

These various results are delineated upon the Diagram accompanying this paper. This shows, in a circle, the number of grains of carbonic acid evolved, and the degree of temperature of the external air on many days in each month of the year. The outer lines show the carbonic acid evolved by myself and Mr. Moul, and the inner line the temperature.

There are, therefore, two seasons of the year in each of which the respiratory phenomena are comparatively uniform for a considerable period, and two others which are truly seasons of change. The former are, in general terms, the winter and summer seasons, and the latter the spring and autumn seasons; and as it will be convenient to give to each of them a short designation, I purpose to speak of them as the maximum, decreasing, minimum, and increasing periods, seasons, and states of system. In order, however, to speak of the subject more accurately, it is neces. sary to refer to shorter periods than three months, and to arrange the months as follows: 
Fixed.

Variable.

Maximum-Jan.,Feb., March, April, May. Decreasing-June.

Minimum -July, Aug., Sept. (part of). $\quad$ Increasing-Oct., Nov., Dec.

In some persons, and probably in some seasons also, May and September must be included in the variable, and December in the fixed.

Thus there is a cycle of changes proceeding from month to month, having definite periods of maximum and minimum intensity, and of increase and decrease, but doubtless varying within limits in different persons, and under the varying degrees of power of the causes to which they are due. These causes are certainly, in great part, the external influences which constitute the seasons; but it is quite possible that there is a law implanted in our nature which in other part governs these changes, and which we shall not be able to refer to the effects of external agencies. Certain it is that the meteorological influences which are associated with the season are insufficient to fully explain the changes which have been described, as the following statement will prove.

Relation to physical phenomena.-The relation of these changes to temperature is not precisely that which has been hitherto received. With sudden accession of heat there was a sudden diminution of the respiratory changes, but in such a manner that, with increasing temperature, the respiration fell not in the same, but in an increasing ratio. With subsequent diminution of temperature the respiration suddenly rose. Hence, in both directions, whether tending to health or disease, the change was disproportionately powerful, in accordance with common observation. This was the most marked in the first great and temporary increase of temperature after the long-continued cold of the winter; and in subsequent accessions during the period of increasing temperature, whilst the direction of the effect was always the same, the degree of it diminished. A medium temperature, such as that at which we endeavour XLII. 
to keep our rooms throughout the year (which is, therefore, the most agreeable to our system in this climate), and such as is found in the open air at three of the four seasons of the year, viz., from $55^{\circ}$ to $60^{\circ}$, is accompanied by all the conditions of the respiration, and which varied in myself from $9 \cdot 13 \mathrm{grs}$. to $6 \cdot 76 \mathrm{grs}$. of carb. acid per minute. Hence, in that degree, temperature and respiration do not maintain any fixed relation. Above $60^{\circ}$ temperature had a more decided influence; but, whatever was the degree of heat, as the season advanced towards August and the beginning of September, the respiratory changes decreased month by month. Thus it is clear that numbers of degrees of temperature bear only a very indefinite relation to the respiratory changes. It is also the fact that the system may become accustomed to the continued influence of a given temperature, and then will exhibit the effect of temperature chiefly at the first sudden change.

The effect of barometric pressure was very similar to that of temperature. The barometer was the lowest at the period of maximum respiration; but a medium height, as between 29 and 30 inches, was accompanied by every variation in the respiratory changes.

The degree of saturation of the atmosphere with vapour had but a very general correspondence with the results obtained from the respiration, but with low temperature and high respiration the degree of saturation was greater, and the contrary with high temperature and low respiration. As, however, the total quantity of vapour contained in the air inspired under the latter circumstances was much greater than with the low temperature, the quantity which could be removed from the lungs by expiration was lessened; and this diminution was further increased by the greatly lessened rate of respiration and the quantity of air inspired.

A review of these three agencies cannot fail to show that the influence of season is much more than can be ascribed to them. There are two other agents which are known to be powerful, but with which we are too imper- 
fectly acquainted to enable me at present to apply them to this subject, viz., light and magnetism. But without hazarding any conjectures as to the precise agencies to which the influence of season should be attributed, there can be no doubt of the truth of the fact which I desire to prove to the Society, viz., that, as the season advances towards autumn, all the respiratory phenomena are lessened, and that they do not reassume an upward tendency until long after the maximum period of temperature has passed over.

Relation to the production and cure of disease.-Such are the facts obtained by me in reference to the respiratory changes; and before I apply them to the production of disease I would meet two objections which would doubtless be urged against their application, viz., that the determination of the amount of carbon emitted by the lungs does not include all the carbon which passes out of the body; and, whatever may be the variations in reference to the carbon, it does not follow that there are similar variations in the plastic changes of the system.

In reference to the first, I reply, that I have, in a certain degree, determined the amount of carbon emitted by the skin (the organ which is loosely believed to act to some extent as vicarious of the lungs), and I find that in July, viz., at a period approaching the minimum period of the respiration, the amount of carbonic acid exhaled per hour by my skin did not exceed six grains-a quantity so disproportionately small when compared with the 500 to 600 grains exhaled per hour by the lungs, that, for practical purposes, in health, the skin cannot be regarded as vicarious of the lungs in reference to chemical changes.

This experiment was made by inclosing the whole body, except the head, in a caoutchouc dress, and passing a known volume of air through the bag so as to maintain the skin in its normal state, the whole air being made subsequently to traverse the analytical apparatus. In reference to the other excretions of carbon and also of nitrogen, I quote the 
results obtained by Barrall in 1848 and 1849. He ascertained during five days in winter, and again in summer, the exact composition and quantity of his food and the composition of his excretions during the period of inquiry, and he found that there was great difference in all the results at the two periods of the year, and in such a manner that the total amount of both carbon and nitrogen in the excretions was lessened in summer, and lessened somewhat proportionately both to the food and to the carbon expired in respiration. Hence, for my purpose, when $I$ prove that the respiratory changes are varied with season, I have a right to say that the vital actions in the system change in like manner. In Barrall's experiments the diminution in the carbon excreted per day, exclusive of that by the lungs and skin; was from 470 grains to 364 grains; and of nitrogen from 210 grains to 182 grains. The variation in the amount of food taken also supports the above assertion.

Thus, the variations in the system, in the summer, may be tabulated as follows :

1. Lessened respiratory and other chemical changes.

2. Usually increased rate of pulsation with increase of blood at the superficies, as shown by the tightness of certain articles of clothing and the colour of the skin; and, as a consequence, a diminished quantity remaining at the centres.

3. Increase in the function of the skin, viz., the transpiration of vapour, and increased sensibility to cold.

4. Lessened difficulty in maintaining a sufficient degree of animal heat, but greater difficulty in preventing excess.

5. Lessened appetite and lessened quantity of food, as proved by Barrall's experiments, in whom the diminution was 28 per cent. in the food taken, and 23 per cent. in the carbon excreted, apart from the lungs and skin.

6. There must be changes in the state of the blood, in reference to the degree of oxidation, the quantity of fibrin, 
and the degree of alkalinity; but these have not been sufficiently inquired into. liver.

7. It is probable that there is increased action of the

Such and so great being the variations in the state of the system, it is in the highest degree probable that they will have a special tendency to the induction of disease. It has long been a familiar fact that the human system is subjected to diseases varying in kind, intensity, and prevalency, with periods of the year. The cause of this has hitherto been sought for out of the system, viz., in the meteorological conditions of the season; but it will now be more proper to connect them with general states of the system due, doubtless, in a great degree, to those meteorological variations.

The dangers to which the body is peculiarly exposed by these variations in its vital actions are of two kinds.

1st. Those which result from the absolute degree of vital action in such a manner that the highest state may be regarded as indicating, or tending to, excess; and the lowest state a defect, or a tendency thereto, in the vital action-states known familiarly as sthenic and asthenic respectively. These dangers have reference to the fixed periods, and will increase with the duration of those pericds.

2dly. Those which result from a want of sufficiently ready adaptation in the processes of the system to the sudden and varying influences from without; as, for example, those of temperature and food. These are peculiarly the dangers of the variable periods, and will be the greatest at the commencement of the variation, or when the changes are unusually violent or rapid.

If it be objected in reference to the former that the lower state of the functions is no evidence of lessened vitality or lessened amount of health, since with it there is a less amount of food taken and less exertion made, and thereby a due balance is still maintained, I would remark -the sense of languor and relaxation, with great heat and 
lessened respiratory and other changes, lessened power of mental application and muscular exertion, special liability to the most destructive diseases at that period, the rapidity with which the inhabitants of hot climates succumb to disease, the increased liability to the attacks of disease when the system is enfeebled and the vital functions low, and vice vers $\hat{a}$; and the greater amount of chemico-vital action in robust and healthy personsall show that health in its two chief characteristics, viz., the power to resist external influences and the capability of the muscles to fulfil their functions, is lessened; and that, in fact, the term "asthenic" and the relative opposite term "sthenic" are applicable to this and the opposite state of the system. This appears to me to be a consideration of great consequence in the application of the effects of season to the production of disease, and if admitted will materially aid us in the determination of the vexed question as to the type of certain diseases.

I have found it of interest to compare these states of system at the different seasons and the dangers just pointed out with the mortality returns of a few diseases which have reference to this subject. It would have been more satisfactory if I could have obtained evidence of admitted value as to the true prevalence of disease; but, unfortunately, the admirable paper of Dr. Guy and the returns to the Board of Health still leave this important branch of knowledge too imperfect to permit me to rely upon it. In taking the mortality returns, however, whilst I shall lose some part of the value of the argument, I shall not import anything which is likely to lead to erroneous conclusions. I have selected the five years from 1850 to 1854, both inclusive, because, during that period, there was not such an amount of variation in the deaths as would disturb the averages-a circumstance which must have resulted if cholera or other epidemic years had been included.

Bearing in mind the four designations already given to the seasons in relation to the vital actions of the system, 
viz., maximum, decreasing, minimum, and increasing, in their order, we find (in the accompanying table) that, in the average of the five years, the deaths from diarrhoa were 36.4 per cent. more numerous in the minimum period than would have occurred if they had been equally distributed over the year. Gastritis and enteritis-and to them may be added nephritis-diseases belonging to a class usually regarded as sthenic in its type, were each about 4 per cent. in excess at the same period. On the contrary, smallpox, measles, pericarditis, bronchitis, pneumonia, pleuritis, and peritonitis corresponded in intensity with the maximum period, and in the minimum period had so great a defect as from 4 to 14 per cent. Certain other diseases are not so restricted in their excess of mortality, but invade the variable periods with or without one of the fixed periods just mentioned. Thus, apoplexy is the most fatal in the increasing and maximum periods. Scarlatina has its greatest excess (12.5 per cent.) in the increasing periodthat is, following the minimum period-and its greatest defect ( 8.3 per cent.) in the succeeding or maximum period; and in both respects typhus corresponds with it, whilst measles is as much in excess $(6.4$ per cent.) in the decreasing-that is, after the maximum period-as it is in defect (5.8 per cent.) in the succeeding or minimum period. Convulsions have their excess in the maximum, and defect in the minimum, periods.

In reference to epidemics-diseases admitted to be usually or entirely seasonal-it is to be remarked that yellow fever, and other virulent diseases of hot climates, are restricted to the minimum period. The plagues in London in $1593,1603,1625$, and 1665, whatever was their nature, began soon after the minimum period had set in, attained the maximum in that period, and terminated in the first month of the increasing period. In like manner all the outbreaks of cholera in this country have invariably attained their great intensity in the minimum period; and although the disease existed, it made no ravages before or after it. 
Influenza, on the other hand, when epidemic in 1847, had its maximum in the increasing and maximum periods.

Table showing the variation per cent. in the mortality from certain diseases in each quarter, on the average of the five years 1850-4, from that which would have occurred if the deaths had been uniformly distributed over all the quarters of the year.

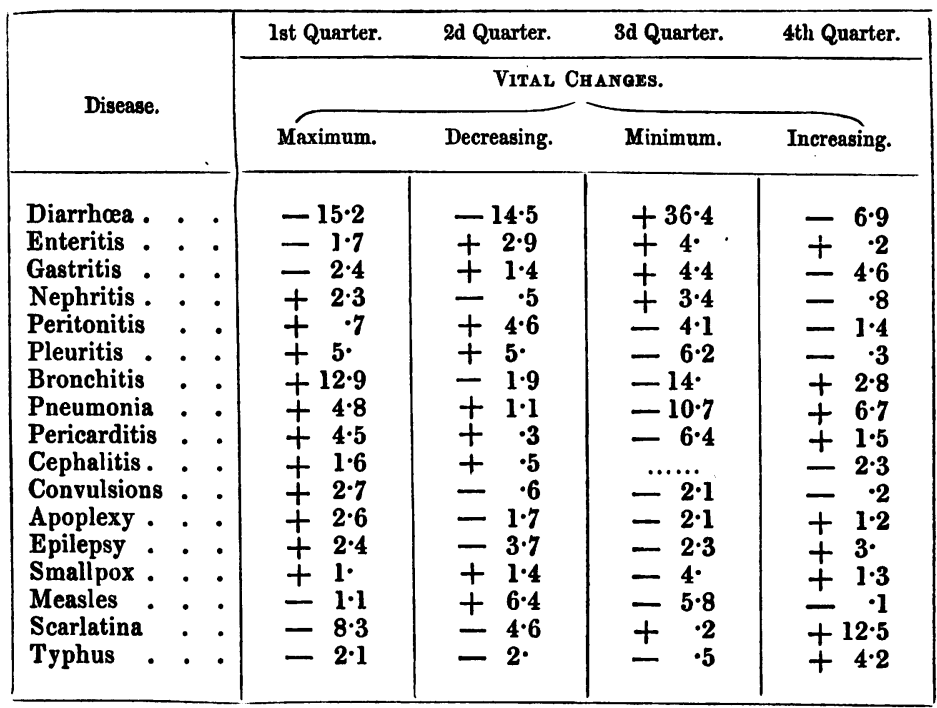

The relation which these diseases bear to each other in their understood sthenic and asthenic types, and with the states of the system now under illustration, is not without interest and importance. Thus there is the widest distinction between those affecting the bowels and the lungs. Cholera, diarrhœa, enteritis, gastritis, and epidemic fevers implicating the bowels, attain the maximum at the minimum period; whilst bronchitis, pneumonia, and pleuritis select the maximum period; and to the latter may be added the allied disease pericarditis, and also convulsions. There is also as marked a difference in the period when these diseases 
begin to prevail. Scarlatina, typhus, apoplexy, epilepsy, convulsions, pericarditis, bronchitis, pneumonia, and pleuritis all increase at the increasing period; whilst measles, gastritis, and enteritis increase with the decreasing period. Cholera, the plague, diarrhœa, and yellow fever are so particularly restricted to the minimum period, that they can scarcely be said to receive their impetus in the variable periods; but if they do so, it is entirely as the vital changes are decreasing. The similarity under this head between scarlatina and typhus, and the dissimilarity between measles and scarlatina, well support the received opinion as to their several types. When these eruptive diseases greatly prevailed there was some difference, so that in the scarlatina of 1844 and 1848 there was a retrocession, and the most fatal periods were the minimum and increasing periods; and in 1845 measles were very prevalent at the same periods. Nephritis was more fatal at both variable periods, and so indeed was peritonitis, but the most so in the decreasing period.

Hence, as a general expression, it may be stated that at the fixed periods diseases of the bowels (minimum) and lungs (maximum) were the most fatal; whilst at the variable periods eruptive and kidney diseases prevailed in the different directions above mentioned.

The deaths from disease of the liver have also their maximum at the minimum period; but the difference with the season is not so marked in this country as to enable me to make use of this fact.

Deductions in reference to disease.-There are four deductions in view of this relation between prevalence and type of disease, and the state of the system, to which I desire to draw attention.

1. It is an admitted fact that the type of the same disease differs in different years, and we know that years vary in the character of their seasons, but into this question I do not purpose to enter. It is also admitted that the type of the same disease varies with the season, so that we look for inflammatory complications in one season and exhaustion in 
the other. It is, I believe, admitted that, cateris paribus, the same disease must be treated differently in different seasons. That certain diseases infest certain seasons chiefly or exclusively has just been shown, and universal experience has proved that diseases so restricted to the minimum period most rarely suffer depletory treatment. To sustain is the aim of the practitioner at that period. Hence, without entering into further detail, I think it may be affirmed that seasonal diseases depend more or less directly upon the degree of vital action existing at that period. This does not preclude the fashionable search after poisons as the cause of the disease ; but it affords a rational and yearly occurring cause within the system for a yearly occurring disease or class of diseases.

The occurrence of a seasonal disease at other periods than its own proper season is easily understood, when we recollect the asthenic condition of so large a part of any community.

2. The type of a seasonal disease will vary according to the direction of the advancing season. Thus, an epidemic of measles occurring in the maximum or the decreasing period of the year, will not tend to inflammatory manifestations, as time advances, but the contrary, since the vital actious of the system are at that period diminishing. On the contrary, an epidemic of scarlatina at the increasing period might have at first presented a marked asthenic type, but with increase of the vital actions this is reduced, and sthenic complications become more apparent. So, it may be inferred, is the natural progress of any disease in which new cases arise over a lengthened period-the advaucing type will be that of the advancing season. These facts are, I think, borne out in practice; but we must be careful not to form an opinion from an isolated case. Hence, in the treatment of seasonal, or indeed of any disease, it is important to bear in mind both the present and the advancing season.

3. Does not this discovery of the varying states of the human system in cycles help us to a rational explanation (at least in great part) of the cessation of seasonal diseases? 
From our want of knowledge, we have been accustomed to say that the disease has worn itself out, or that, having carried off its victims, there is no more food for the destroyer, without our ever presuming that such explanations were satisfactory. But if an epidemic arises from, or only in, a certain state of system, it may increase as that condition of system increases; but it must decline when, by a further continuance, that condition of system is changing into its opposite. Thus, cholera or diarrhœa may appear in isolated cases at or before the beginning of June; but as that month advances, and the vital actions are decreasing, it bursts out with violence, and continues to increase until the middle of August, when the system has been attaining its lowest state of activity or vitality; but in September the deaths decline, and in October they cease-ceasing at a period when the system has assumed its upward tendency. Such, at least, has been the experience in our attacks of cholera, and such is now proved to be the progression in the vital actions. Only in this way can we account for the great excess of scarlatina in the fourth quarter, and its great defect in the following quarter. It is now well understood that, whatever inflammatory or febrile aspect it may assume, it is essentially a disease of the changing season, and is not sthenic in its character. Immediately the system has regained its maximum amount of vital power, it ceases to give development to scarlatina. As has been already shown, measles succeed to the maximum state, as scarlatina succeeded to the minimum state, and loses its sthenic character as the summer advances. Both diseases are most severe in the early part of the outbreak-the one by a tendency to exhaustion, the other to inflammation.

4. I venture also to suggest that this cyclical rotation in the variation of the vital powers is one of the explanations of the "Vis medicatrix naturæ." That there is a tendency implanted in the system to the cure of disease is undoubted; and as disease is commonly an exaggeration of the natural tendency of the system-that tendency varving as we have seen in direction and intensity in a defined and consecutive manner - a period must arrive when the 
diseased state shall be placed under curative conditions, and the system which induced the disease shall become its own physician. This explanation may not be applicable to the cure of acute disease, but $I$ deem it to be quite applicable to various states of the system not entirely dependent upon important structural changes.

In conclusion, it has been shown that whilst, in accordance with the statements of all observers, the respiratory function is ever varying, the changes pursue a definite cycle through the day and through the year. These two cycles are inseparably connected, for when that of the day is completed it does not always again start from the starting-point of the previous day, but assumes a new direction induced by the cycle of the seasons, so that the absolute quantities represented by the former are increased or decreased in a definite manner by the latter.

If there be truth in the observations now presented to the Society, we shall readily perceive that, however much the influence of seasons may induce disease, the succession of the seasons and their cyclical return is of the utmost value to man; and they give us another link in that great chain which binds the animal and vegetable creations together as one undivided whole. They also expose the folly of striving to maintain one unvarying season by artificial heat in winter and timid exposure in summer-an evil probably less prevalent now than in former years. They further show the propriety of freely exposing the body to the full influence of the ordinary changes of the seasons - a plan which, in the treatment of such a disease as phthisis, has effected an incalculable amount of good-as well as of protecting the system from the effect of seasons of undue severity or duration.

After a lengthened consideration of the subject, I am led to believe in the truthfulness of the views now given, and to hope that the demonstration of the seasonal changes of the system may lead to new ideas, and that some of the deductions now given contain the germ of fundamental truths. 
Postscript.-The influence of season on the viability of children.-Since writing the foregoing paper, it has occurred to me to ascertain if the season of the year at which children are procreated or born affects their viability, or their subsequent liability to diseases of a type similar to that of the state of the human system existing at the season of their birth. By the kind permission of the Registrar.General and Dr. Farr, I have abstracted all the deaths of children dying in the northern district, from Newcastle to Kendall, in 1857 (a non-epidemic year), whose age in months was recorded with their death, and have thereby ascertained the period of their birth. The total number is 3050, nearly all of whom were under 1 year of age; and although the number is not very large, it is, in Dr. Farr's opinion, sufficient to reason upon. The result shows that of these children the largest per centage was born in June; and then in May, July, and August; whilst, after the latter month until January, the per centage was below the average. These months of diminished viability are those with which the human system has been shown to be in a declining or minimum state of vitality, and the effect is associated rather with the period of birth than the period of conception. The period is not that at which the greatest number of births occur. From this circumstance, as well as from the fact that the sexual appetites of animals have been so arranged that they are the most excited in the summer season (a period of year different from that of the birth of their offspring), it appears highly probable that the young differ in viability with the season of their birth, and therefore with the vital changes proceeding in their system at the period of their birth. This is also supported by the well-known fact of the influence of the health and constitution of the parent over the viability of the offspring.

I have commenced an inquiry as to the influence of the season of year at the time of birth over the tendency to the induction of certain classes of diseases, but it is still incomplete. 
110 CYClical Changes in THE hUMAN SYSTEM.

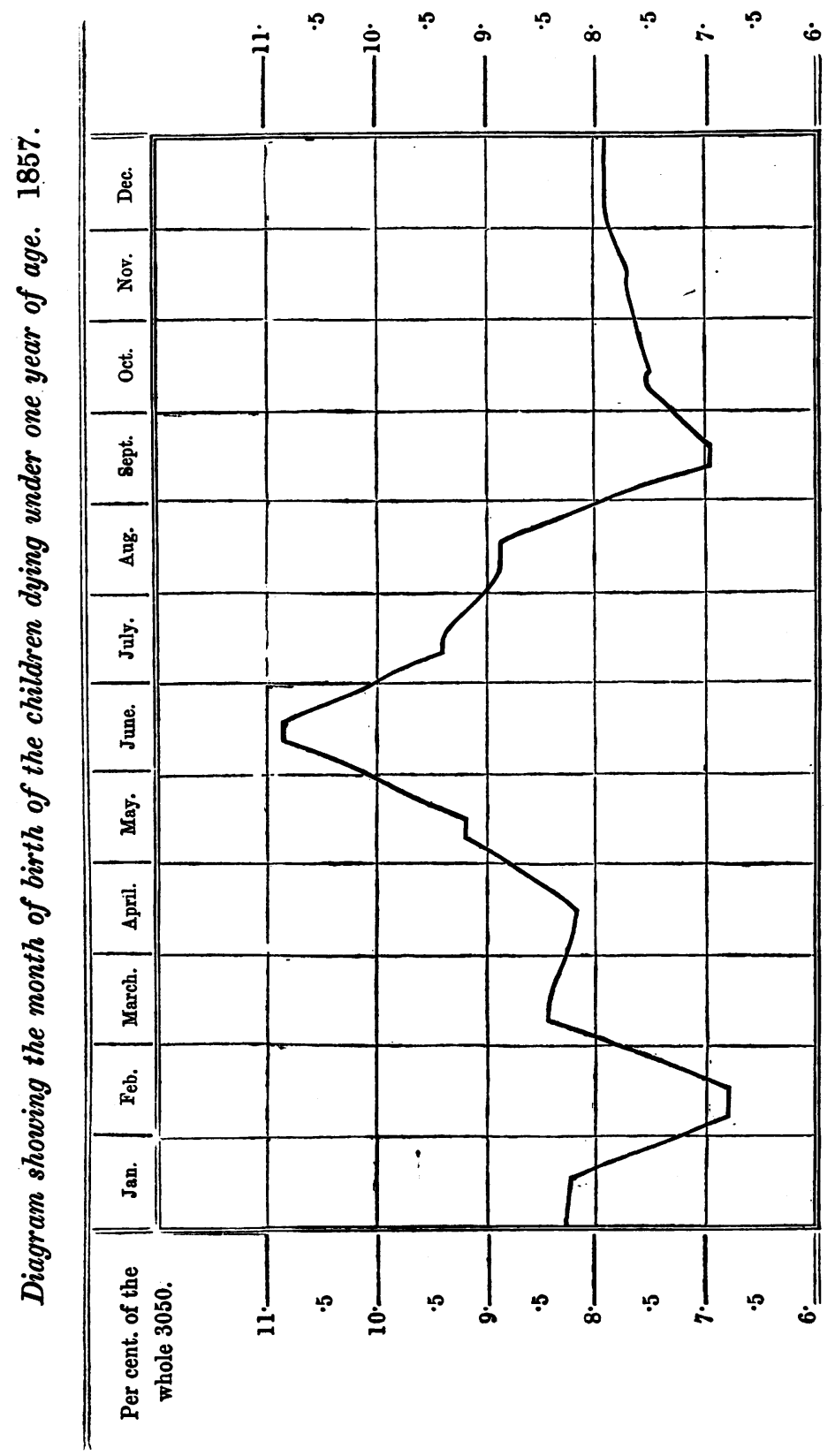

